\title{
Cyber physical system for vehicle counting and emission monitoring
}

\author{
Ali Khan ${ }^{1}$, Khurram S. Khattak ${ }^{2 *}$, Zawar H. Khan ${ }^{3}$, Mushtaq A. Khan' ${ }^{1}$ and Nasru Minallah ${ }^{2}$ \\ Research Assistant, National Centre for Big Data and Cloud Computing, University of Engineering and Technology \\ Peshawar, Pakistan ${ }^{1}$ \\ Assistant Professor, Department of Computer Systems Engineering, University of Engineering and Technology \\ Peshawar, Pakistan ${ }^{2}$ \\ Assistant Professor with the Department of Electrical Engineering, University of Engineering and Technology \\ Peshawar, Pakistan ${ }^{3}$ \\ Received: 04-August-2020; Revised: 22-September-2020; Accepted: 25-September-2020 \\ (C)2020 Ali Khan et al. This is an open access article distributed under the Creative Commons Attribution (CC BY) License, \\ which permits unrestricted use, distribution, and reproduction in any medium, provided the original work is properly cited.
}

\begin{abstract}
Transportation with $29 \%$ share in overall greenhouse gas emissions, is a major source of urban health and environmental degradation. A lot of effort has gone into development of different solutions to analyse, control and manage traffic flow to reduce vehicular emissions. In this regard, a low-cost, easily installable and maintainable solution for traffic flow characterization is of utmost importance to provide true intelligent transportation solutions. In this work, a raspberry pi based cyber physical system has been proposed for vehicle counting using image processing. Moreover, the proposed solution has the capability to measure associated roadside vehicular emissions such as carbon dioxide, carbon monoxide and particulate matter. The proposed solution can be used to develop relationships between traffic flow and associated roadside pollutants. For data logging and analytics, the sensed parameters were transmitted to a free and open source cloud platform "ThingSpeak". For field testing, the proposed solution was installed on a main thoroughfare in 42 minutes. Sensed parameters were transmitted per minute with $100 \%$ accuracy to ThingSpeak using Wi-Fi. Vehicle counting accuracy of the proposed system was $86.9 \%$. On-road traffic flow was successfully characterized in terms of traffic flow, density and average time headway. Relationships between measured traffic flow parameters and associated sensed pollutants (carbon dioxide, carbon monoxide and particulate matter) were established. The proposed solution to the fabrication cost of $\$ 70$ has the capability to operate for 13 hours without any human intervention.
\end{abstract}

\section{Keywords}

Intelligent transportation system, Raspberry Pi, ThingSpeak, Vehicle count, Vehicle emissions.

\section{Introduction}

With $25 \%$ share in world's fossil fuel consumption, the transportation sector is contributing about $29 \%$ of worldwide greenhouse gas (GHG) emissions [1]. Furthermore, about $23 \%$ of global carbon dioxide (CO2) emissions are attributed to the transportation sector [2]. Other transportation related pollutants include nitric oxides, sulphur dioxide and particulate matter (PM) dust in urban settings. According to WHO report, transportation related ambient air pollution has resulted in 4.2 million premature deaths worldwide in 2016 [3]. About $91 \%$ of these deaths are born by low and middle-income countries [3]. With increasing urbanization, traffic related health issues will exasperate further, resulting in increased lung inflammation, cardiovascular, pulmonary and respiratory diseases [3-5].

*Author for correspondence

181
Hence it has become imperative to devise strategies for better urban mobility management. This can either be achieved by enlarging urban road infrastructure and building new access roads with extremely high costs. Or it can be achieved through implementation of intelligent transportation system (ITS) methodologies. For ITS based solutions, detailed traffic flow parameters (such as vehicle count, classification, speed, time headway, road density) are fundamental building blocks. Furthermore, these traffic flow parameters are instrumental for better planning, designing and management of road networks [6, 7].

In existing literature, different solutions have been proposed for traffic flow characterization and are generally categorized either as intrusive or nonintrusive sensors [8]. However, both intrusive sensors (such as inductive loop, pneumatic tube, piezoelectric 
and magnetic sensors) and non-intrusive sensors (such as accelerometer, acoustic, ultrasonic, radar, $\mathrm{Wi}-\mathrm{Fi}$ ) have their own limitations which limit their applicability under varying traffic conditions. These limitations range from their incapability to characterize traffic flow under congested and heterogeneous traffic behaviour. Furthermore, both intrusive and non-intrusive sensors are incapable of providing full range of traffic flow parameters. Meteorological conditions (such as rain, wind, sunlight, fog, temperature) further affect the accuracy of both types of aforementioned sensors.

In this context, compute vision-based edge computing solutions are emerging as an optimum solution for traffic flow characterization. Though, running compute heavy image processing algorithms under the constraint of compute resource is a major hurdle in providing edge computing solutions. For this reason, Raspberry $\mathrm{Pi}(\mathrm{RPi})$ based edge computing solutions for traffic flow characterization are limited in existing literature [9]. Thus, providing ample room for innovation to employ low-cost RPi for traffic flow characterization.

With this perspective, a RPi based cyber physical system has been proposed in this work. The proposed solution has the capability to count vehicles and measure associated roadside vehicular pollutants such as carbon dioxide (CO2), carbon monoxide $(\mathrm{CO})$ and particulate matter (PM2.5). Vehicle count is measured using compute vision algorithms running on RPi. The sensed data is transmitted through ESP8266 Wi-Fi module to a free and open source cloud platform "ThingSpeak" for logging, processing and analysis. Sensed parameters were transmitted per minute ThingSpeak with $100 \%$ accuracy. Vehicle count accuracy of the proposed system was $86.9 \%$ in field testing.

Main objective of this undertaking is as following:

- Provide a low-cost, real time cyber physical solution for traffic flow characterization,

- Employing a free and open source cloud platform for data logging and analytics.

- Establishing relationships between traffic flow parameters (such as vehicle count, density, time headway) and roadside traffic pollutants (such as $\mathrm{CO}, \mathrm{CO}_{2}$ and $\mathrm{PM}_{2.5}$ )

Rest of the paper is organized such that Section II discusses related work. Section III details system architecture of the proposed system while section IV presents results. Discussion and Conclusion are detailed in section V and VI respectively.

\section{Related work}

In existing literature, limited solutions have been proposed for traffic flow characterization using low cost compute vision based embedded devices. However, in the recent past, RPi based solutions for traffic flow characterization have emerged as an optimum solution because of its low cost. These solutions range from estimating vehicle count [10, $11]$, vehicle count and classification [4, 12, 13] or vehicle count and speed estimation $[8,9,14]$.

\subsection{Counting}

Wiska at el. [11] proposed RPi based solution for night-time vehicle counting through vehicle's headlamps. The authors achieved high counting accuracy based on symmetry between headlights. Furthermore, the same vehicle counting algorithm was run on four different embedded devices such as RPi B+, Beagle board Xm, RPi 2 and Odroid XU4 for comparison purposes. An accuracy of $92.6 \%$ was achieved on Odroid XU4 because of the former's higher computation power. Anandhalli et al. [10] proposed a solution for vehicle counting using RPi and OpenCV. Vehicle counting accuracy of $96 \%$ was reported from top and side view, 92.8\% from front and rear view, and 93\% from multi view. Vehicle's colour features were extracted to distinguish vehicles from background. Vehicle's contour was located by converting images from RGB to HSV, tracking vehicles through their calculated centroid points using Kalman filters. The time to process one frame was reported at $81.5 \mathrm{~ms}$.

\subsection{Counting and classification}

In [12], an RPi based solution was proposed for vehicle counting and classification (either as car or bus). The proposed system had the capability of working in different lightning conditions such as day and night. The image processing technique worked by cropping unnecessary portions, detecting bright and dark vehicles with maxima and minima transform and performing morphological operation to identify connected vehicles. An accuracy of $91.08 \%$ and $97.47 \%$ was reported in medium and heavy traffic conditions. During night time, an accuracy of $88.16 \%$ was reported. Gregor et al. [4] proposed an RPi 2 based solution for vehicle counting and classification in real time using OpenCV and $\mathrm{C}++$. To overcome compute resource limitation of $\mathrm{RPi}$, image processing algorithm was divided into four steps to be run in parallel on RPi's four cores. The four different steps were (1) image capturing, (2) background subtraction, (3) detection, and (4) counting and classification. Among these four steps, 
the background subtraction step was reported as most compute heavy. Furthermore, to overcome compute resource constraints, video's resolution and frame per second (fps) were kept to a minimum of $640 \times 360$ and 15 fps respectively. The measured data was first stored in a local database and later transmitted to a remote server using SIM800L GPRS module. In [13], RPi 3 based vehicle counting and classification solution was proposed using OpenCV. The counting accuracy was reported at $83 \%$ under different meteorological conditions (such as sunny, rainy, sunset) and night time. Kalman filters were employed for object tracking, while classification was done using object size characterization in Support Vector Machine (SVM). The measured parameters were transmitted to a remote server using HTTP protocol.

\subsection{Counting and speed estimation}

Iszaidy et al. [8] proposed RPi based vehicle speed estimation solution using OpenCV and Python. Speed was estimated by tracking the vehicle's image pixel coordinates in different frames. Comparison of a video with three different resolutions (320p, 540p, 720p) was undertaken to note the effect of resolution on RPi's CPU and memory utilization. Though CPU utilization was the same for all three, memory utilization of video with $720 \mathrm{p}$ resolution was highest at $600 \mathrm{MB}$. In [9], a solution for real time vehicle counting and speed estimation was proposed using RPi 3. Using OpenCV, image processing techniques were used on a video of $640 \times 480$ resolution and 30 fps. Field testing was conducted and speed accuracy of $91.98 \%$ and $87.5 \%$ were reported in residential areas and freeways respectively. Counting accuracy of $100 \%$ was achieved. In [14], vehicle's count and speed were estimated using Gunnar Farneback method through optical flow calculation from a prerecorded 30 fps video on RPi 3. Using Wi-Fi, sensed parameters were transmitted to a remote server for analysis.

The novelty of proposed solution in this work is not only to measure vehicular flow but also to estimate associated vehicular emissions such as $\mathrm{CO}, \mathrm{CO} 2$ and PM2.5. Relationships between vehicle count and associated vehicular emissions were established. The proposed solution can be employed to:

- Characterize traffic flow at different road sections and estimate associated vehicular pollutants.

- Help traffic engineers to identify congested road sections and affiliated environmental costs.

- Macroscopic vehicular flow parameters can be employed for validation and calibration of mathematical traffic flow models and traffic simulation software [6, 7], [15-18].

Furthermore, a free and open source cloud platform was employed for logging and analysis of data for scalability and reliability.

\section{System architecture}

Traffic related GHG emissions are major source of urban pollution, affecting both quality of life and urbanities' health $[2,3]$. As such, a low-cost real time cyber physical solution has been proposed for vehicle counting and associated vehicular emissions such as $\mathrm{CO}, \mathrm{CO} 2$ and PM2.5. The proposed solution can be employed for measuring road densities and associated environmental costs. The proposed system has been categorized in four modules: (1) Sensor node, (2) Software design, (3) Current consumption, and (4) Cloud platform.

\subsection{Sensor node}

From existing literature, it is evident that the biggest stumbling block in providing a true edge computing solution for real time traffic flow characterization is resource constraint embedded board. For computation at edge location, a low cost and power optimized embedded device with enough computation resources is important. RPi [19] with its compact size, low cost and power optimized computation resources, has emerged as the best choice as evident from existing literature. As such, $\mathrm{RPi} 3 \mathrm{~B}$ with $1.2 \mathrm{GHz}$ processor and $1 \mathrm{~GB}$ RAM is employed in this work. Salient features of RPi 3B are its four ports (USB, Ethernet, CSI (Camera Serial Interface)) and 40 digital GPIO (General Purpose Input Output) pins. Raspbian, an optimized operating system for $\mathrm{RPi}$ devices is running our $\mathrm{RPi} 3 \mathrm{~B}$. OpenCV library installed on RPi is used for vehicle counting using a live video stream of $640 \times 480$ resolution at $20 \mathrm{fps}$. Pi camera connected to $\mathrm{RPi}$ through CSI port is being used for video capturing as can be seen in Figure 1.

Though in existing literature, numerous RPi based edge computing solutions have been proposed for vehicle counting and classification [4], [ 11-13] or vehicle counting and speed estimation [8, 9, 14]. However, the novelty of our proposed solution is that in addition to vehicle counting, it has the capability to measure associated road-side vehicular emissions such as $\mathrm{CO}, \mathrm{CO} 2$ and $\mathrm{PM} 2.5$. Low cost environmental sensors MQ135 (for CO and CO2) and GP2Y1010AU0F optical sensor (for PM2.5) have been integrated to keep the overall cost low. Because of unavailability of analog I/O pins on RPi, the two 
Ali Khan et al.

environmental sensors have been integrated on Arduino Nano as can be seen in Figure 1 . Intercommunication between RPi and Arduino Nano is done through serial communication via USB port.

For time synchronization, RTC has been integrated with Arduino Nano so that traffic flow can be correlated with sensed road-side vehicular pollution. Sensed parameters are transmitted to cloud platform
'ThingSpeak' using ESP8266 Wi-Fi module integrated with Arduino Nano. For reliability purpose, SD card module has been integrated for data logging as can be seen in Figure 1. In case of missed transmitted data to cloud platform because of internet issues, logged data in SD card can be retrieved. Fully fabricated sensor node can be seen in Figure 2.

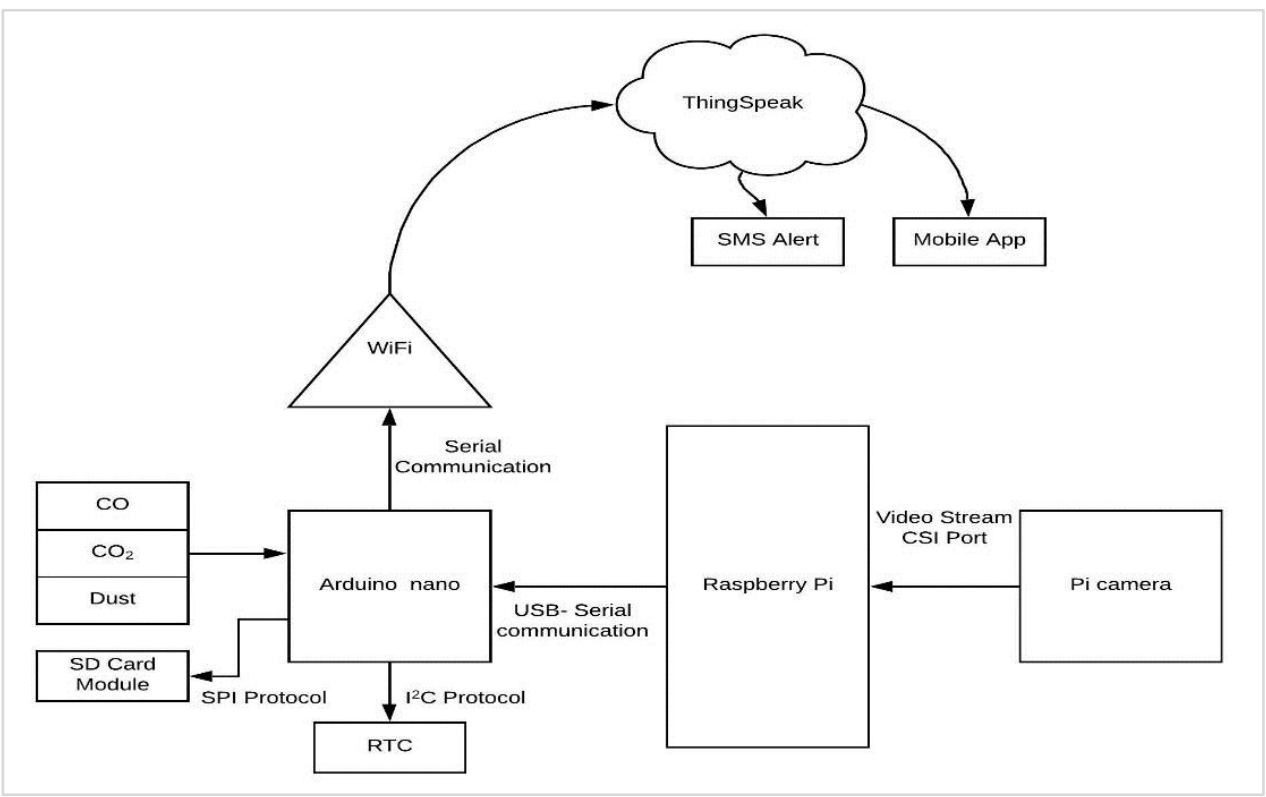

Figure 1 Proposed cyber physical system's design overview

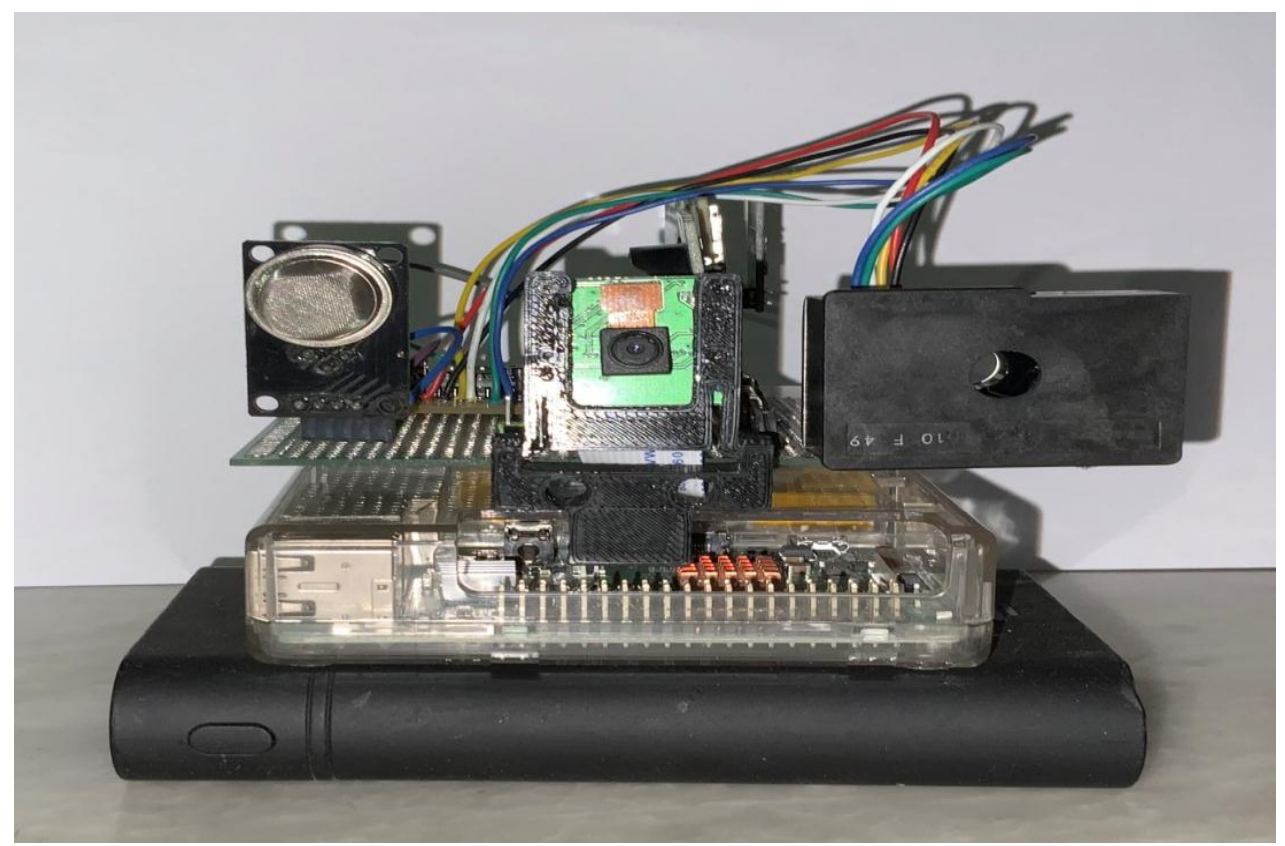

Figure 2 Sensor node with RPi 3B, Pi camera, Power bank and environmental sensors 


\subsection{Software design}

For the software part, the Raspian operating system has been installed on RPi. For image processing OpenCV libraries have been employed using $\mathrm{C}++$ programming language.

OpenCV (Open Source Computer Vision Library) is an open source and free machine learning library for image processing. OpenCV library consists of more than 2500 optimized image processing algorithms such as video capturing and encoding, object tracking and identification, and Hough transform [8]. Using OpenCV algorithms, vehicles are counted in realtime using Pi camera. Deployed software algorithm can be categorized in six steps as given below. Software flow diagram can be observed in Figure 3.

1 Image capturing: Live video stream of $640 \times 480$ resolution at $20 \mathrm{fps}$ is captured through Pi camera attached to RPi through CSI port. Input frames are analysed one by one for further analysis.

2 Background Subtraction: In this step, the foreground is separated from background by subtracting the current frame from the previous one. Output of this step is a binary image showing vehicles as white blobs.

3 Contour Detection: In this step, blob's shape analysis in each frame is performed for better localization.
4 Convex hull: Proper shaping of detected objects is performed in addition to noise removal.

5 Tacking: To avoid repeat counts of the same vehicle, a unique id is assigned to each blob and is tracked till it leaves Region of Interest (ROI).

6 Count: An ROI is drawn in image frames for vehicle counting whenever it crosses it.

7 Vehicle count is relayed to Arduino Nano every 15 sec using USB-Serial communication as can be seen in Figure 1.

8 Vehicular emissions are sensed after getting vehicle count by Arduino Nano.

9 For reliability, measured parameters are stored locally on an SD card integrated with Arduino Nano.

10Measured parameters are transmitted to ThingSpeak via ESP8266 Wi-Fi module every 15 sec.

\subsection{Current consumption}

The proposed system is powered through a Xiomi Mi 10,000 mAh power bank. The power bank comes with dual power output USB ports, providing 5.1/2.4A each. RPi is powered through the power bank. Arduino nano with integrated environmental sensors, SD card and Wi-Fi module is powered through RPi's USB port. Current consumption of individual components is detailed in Table 1.

Table 1 Proposed system's current consumption breakdown

\begin{tabular}{ll}
\hline Modules & Current consumption \\
\hline RPi (LEDs, HDMI and Wi-Fi OFF) & $200 \mathrm{~mA}$ \\
\hline Pi camera & $250 \mathrm{~mA}$ \\
\hline Arduino Nano & $20 \mathrm{~mA}$ \\
\hline MQ-135 $\left(\mathrm{CO}\right.$ and $\left.\mathrm{CO}_{2}\right)$ & $160 \mathrm{~mA}$ \\
\hline GP2Y1010AU0F $\left(\mathrm{PM}_{2.5}\right)$ & $20 \mathrm{~mA}$ \\
\hline Wi-Fi (ESP8266) & $80 \mathrm{~mA}$ \\
\hline SD Card & $0.5 \mathrm{~mA}$ \\
\hline RTC3231 & $0.3 \mathrm{~mA}$ \\
\hline
\end{tabular}

Using values reported in Table 1 and equation (1), current consumption of the proposed system was estimated at $730.5 \mathrm{mAh}$.

$I_{\text {Total }}=I_{R P i}+I_{\text {Pi camera }}+I_{\text {Arduino nano }}+$

$I_{M Q 135}+I_{P M 2.5}+I_{S D \text { card }}+I_{W i-F i}+I_{R T C}$

This was further verified by monitoring current consumption of the proposed system through Keweisi KWS-V20 USB tester. Integrated 10,000 mAh power bank can power the proposed system for 13.68 hours when operational.

\subsection{Cloud platform}

The proposed system was designed to provide a lowcost and real time solution. From this perspective, an open source and free cloud platform 'ThingSpeak' has been used for sensed data storage, processing and analysis. Sensor's node data is transmitted to ThingSpeak through ESP8266 Wi-Fi module using HTTP protocol. The data upload rate is once per minute. Furthermore, ThingSpeak provides various inbuilt apps and functionalities for data analytics and information dissemination. These tools range from SMS and Email alert, data visualization through mobile app and built-in MATLAB tools for custom visualization $[20,21]$. 
Ali Khan et al.

\section{Results}

For field testing, sensor node (as shown in Figure 2) was installed on a pedestrian bridge over a main thoroughfare 'Ring Road' in Peshawar, Pakistan. Sensor node's installation site and camera view can be seen in Figure 4 and 5 . The evaluation time duration was 42 minutes from 4:11 PM to 4:53 PM on Tuesday 14th January, 2020. To evaluate vehicle counting accuracy of the proposed system, video of the same duration, location and time was recorded through a camera. A total of 1109 vehicles were counted by the proposed system as opposed to 1275 vehicles counted manually in the video. Thus, vehicle counting accuracy of the proposed system was noted at $86.9 \%$. Although sensed parameters transmission rate to ThingSpeak is every $15 \mathrm{sec}$, sensed road-side vehicular emission parameters are averaged for one minute as can be seen in Figures 6, 7, and 8. This is to establish clear relationships between vehicle numbers and associated roadside vehicular emissions.

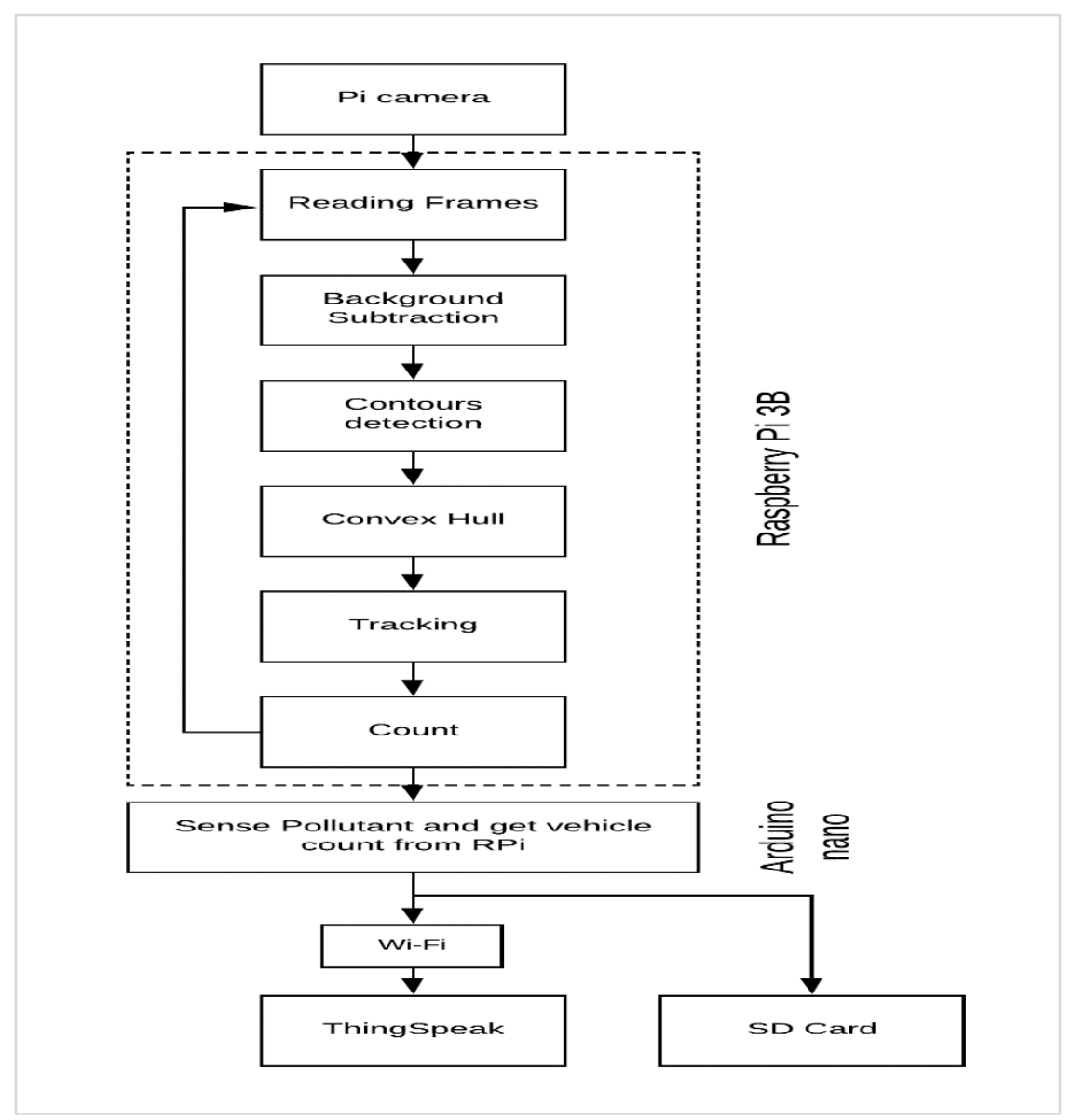

Figure 3 Overview of software flow diagram 
International Journal of Advanced Computer Research, Vol 10(50)

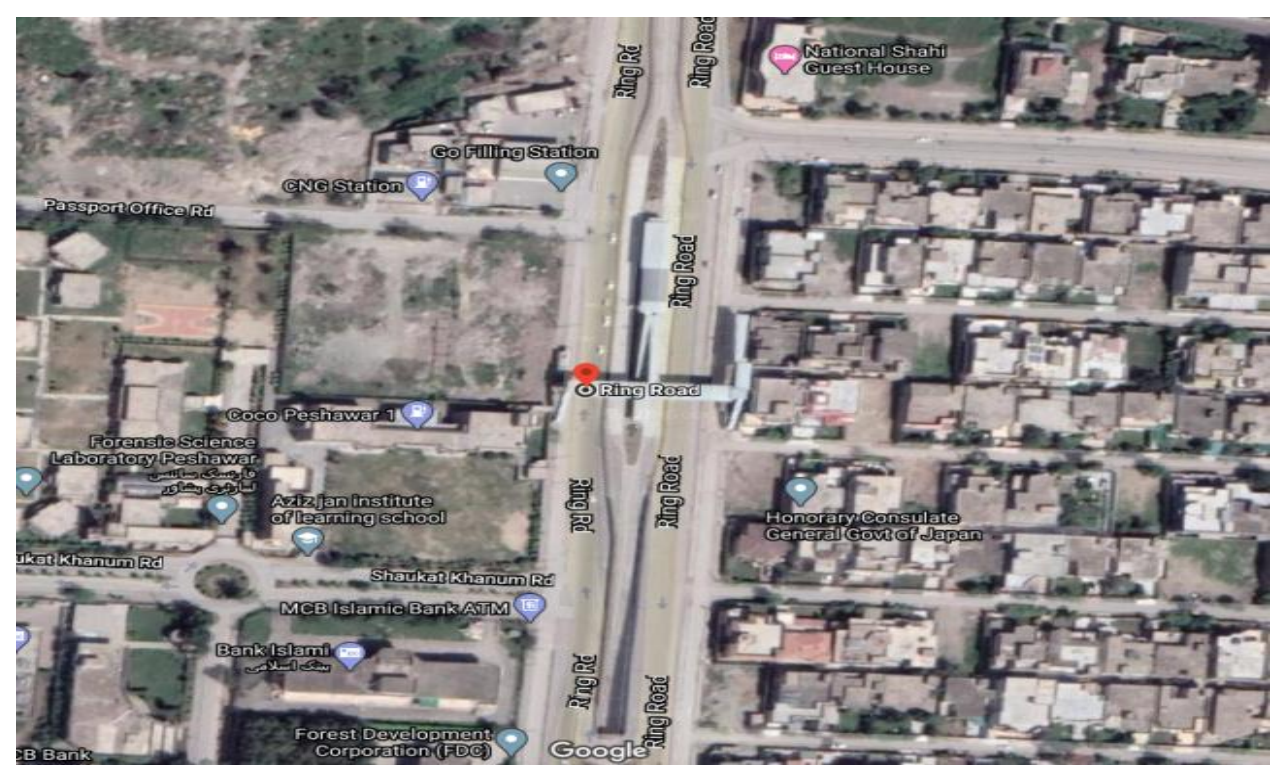

Figure 4 Field testing site 'Ring Road' in Peshawar, Pakistan

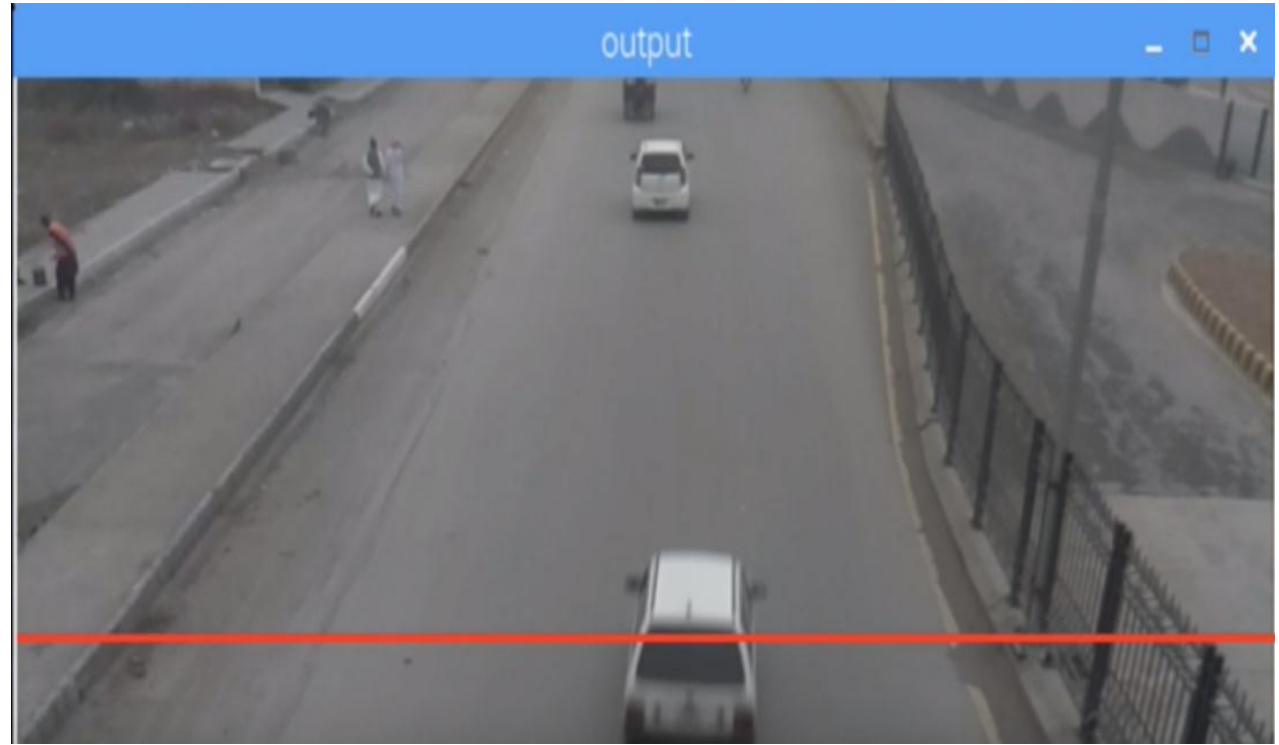

Figure 5 Sample image from video captured through sensor node 
Ali Khan et al.

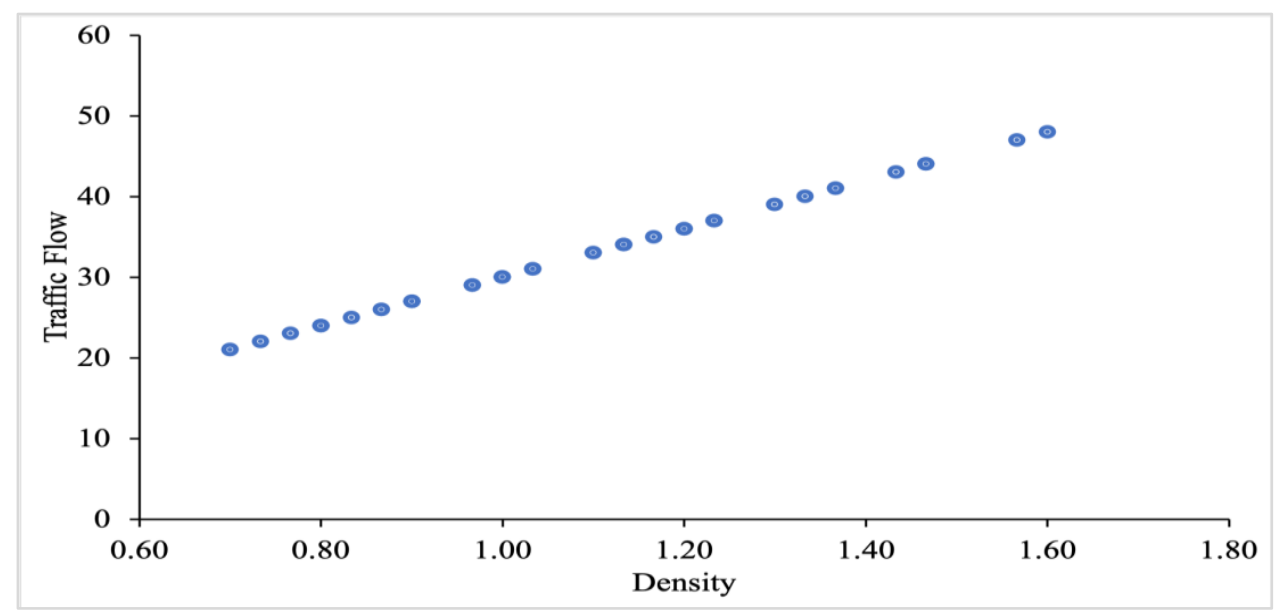

Figure 6 Trend of traffic flow and density over a 42 min period on Tuesday 14th January, 2020

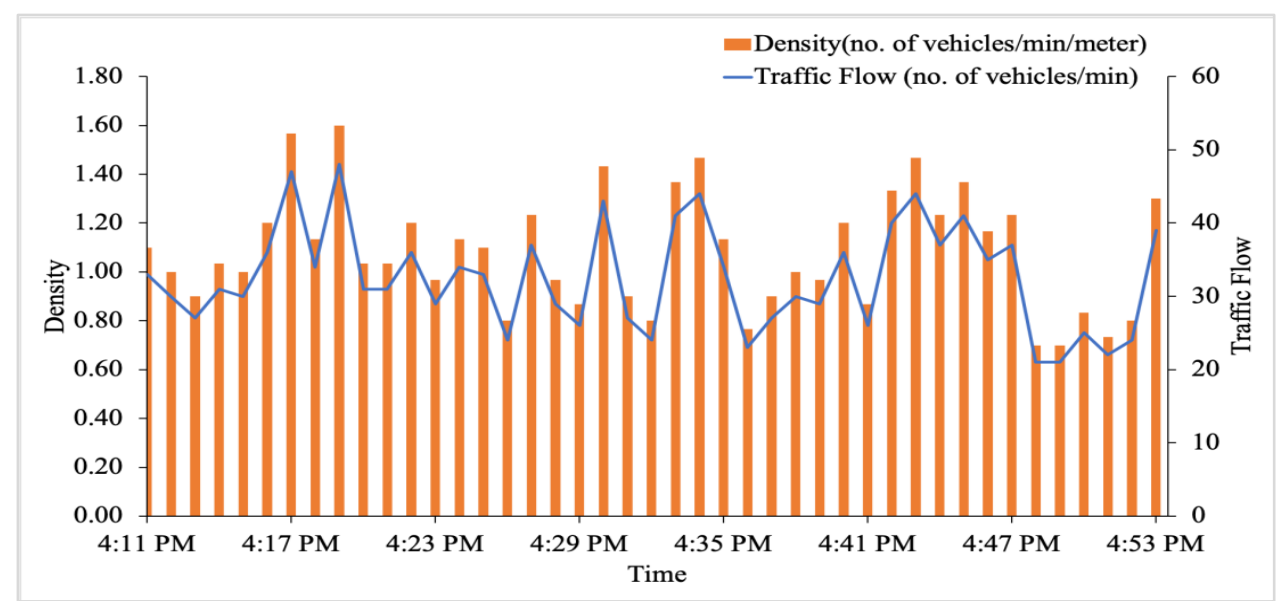

Figure 7 Traffic flow and density evaluation over a 42 min period on Tuesday 14th January, 2020

Macroscopic traffic flow parameters such as traffic density and average velocity have been extracted using measured vehicle count. Road traffic density is

Density $=\frac{\text { Vehicle count }}{\text { unit length of road }}$

While traffic flow is

Traffic flow $=\frac{\text { Vehicle Count }}{\text { minute }}$.

Average time headway is required by a driver to align to the traffic changes noticed, and is calculated from data using equation (4) [22].

Average time headway $=\frac{1}{\text { Traffic flow }}$.

Measured parameters such as traffic flow, density, average time headway, $\mathrm{PM}_{2.5}, \mathrm{CO}$, and $\mathrm{CO}_{2}$ for an evaluation period of $42 \mathrm{~min}$ are tabulated in Table 2.

Traffic flow and density obtained are plotted in Figure 6, which established a direct relationship between them. As traffic flow (number of vehicles/min) increases, density (no. of vehicles $/ \mathrm{min} / \mathrm{m}$ ) will also increase proportionally. In other words, as long as the road density is below critical density, an increase in traffic flow will increase road density.

As can be seen in Figure 7, at 4:16 PM traffic flow is $36 \mathrm{veh} / \mathrm{min}$, while traffic density is $1.20 \mathrm{veh} / \mathrm{min} / \mathrm{m}$. While at 4:17 PM, traffic flow is $47 \mathrm{veh} / \mathrm{min}$, and traffic density has increased proportionally to 1.57 $\mathrm{veh} / \mathrm{min} / \mathrm{m}$.

In Figure 8, the inverse relationship between traffic flow and average time headway has been detailed. Minimum and maximum average time headway were recorded at $1.25 \mathrm{~s}$ and $2.85 \mathrm{~s}$ respectively. As can be seen in Figure 8, maximum traffic flow of 48 $\mathrm{veh} / \mathrm{min}$ was observed at 4:19 PM with an average time headway between vehicles at $1.25 \mathrm{~s}$. While at 4:49 PM, minimum traffic flow of $21 \mathrm{veh} / \mathrm{min}$ was observed with average time headway at $2.85 \mathrm{~s}$. The 
safe headway is $4 \mathrm{~s}$. However, the observed time headway is quite below $4 \mathrm{~s}$, and the traffic is more vulnerable to accidents. This is one of the major causes of stop and go traffic behaviour in Peshawar, Pakistan.

Carbon monoxide though absent in natural settings, results from incomplete combustion of fossil fuels in absence of enough oxygen. As can be observed in Figure 9, sensed CO levels have a direct relationship with on-road vehicle numbers. The lowest level of $\mathrm{CO}$ was recorded at $2 \mathrm{PPM}$ when traffic flow was 20 veh/min. Highest level of $\mathrm{CO}$ was recorded at 4.14 PPM at 4:42 PM, when traffic flow was $40 \mathrm{veh} / \mathrm{min}$ as can be observed from Figure 9 and Table 2. A direct relationship was observed between on-road vehicle numbers and $\mathrm{CO}_{2}$ levels as can be seen in Figure 10. Just like $\mathrm{CO}$ as shown in Figure 9, the peak value for $\mathrm{CO}_{2}$ at 1187 PPM was observed at 4:42 PM. In the observed period, average $\mathrm{CO}_{2}$ levels were recorded at 871 PPM.

The peak of $\mathrm{PM}_{2.5}$ concentration was observed at 99 PPM at 4:42 PM as can be observed in Figure 11 . The lowest concentration of $\mathrm{PM}_{2.5}$ was recorded at 4:13 PM.

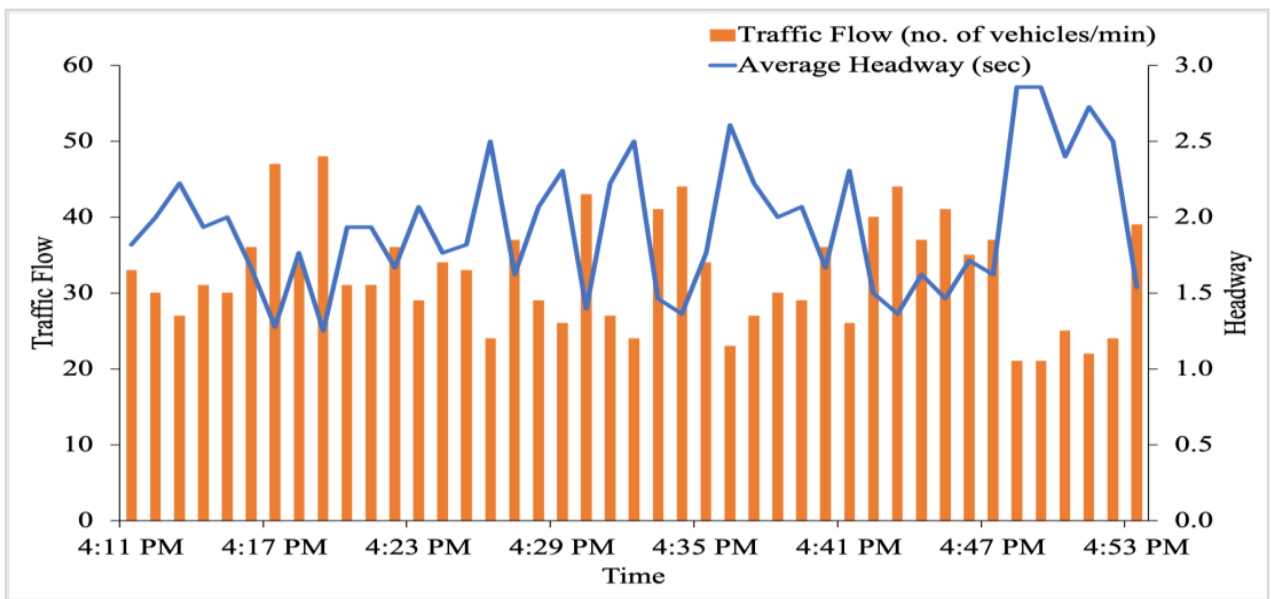

Figure 8 Traffic flow and average time headway over a 42 min evaluation period on Tuesday 14 th January, 2020

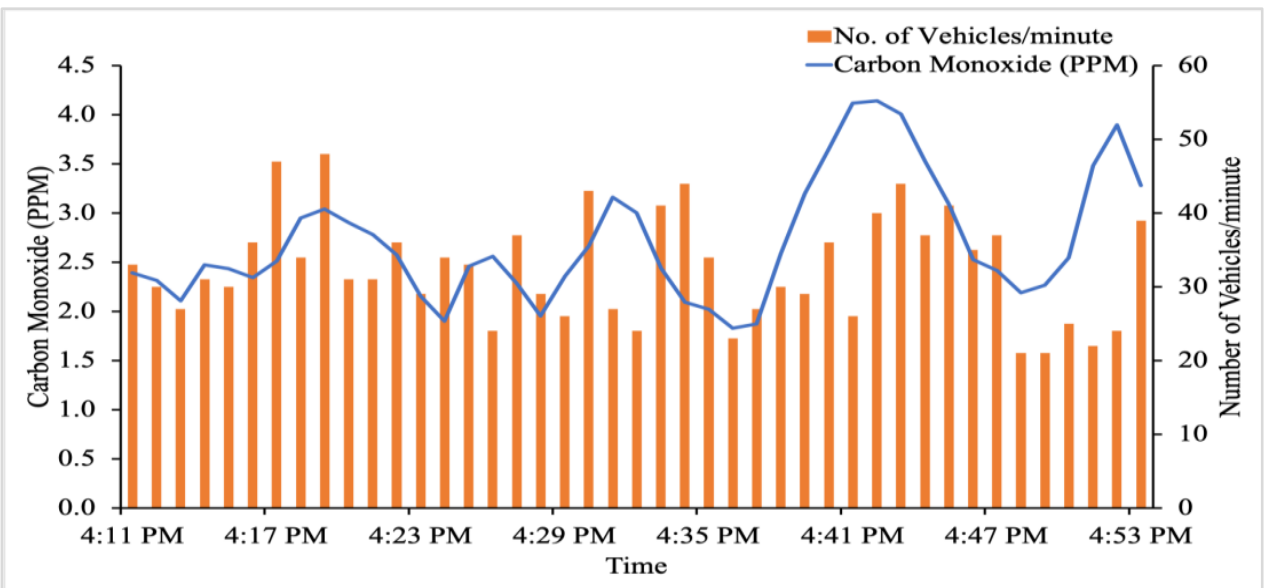

Figure 9 Relationship between Traffic flow and Carbon Monoxide over a 42 min period on Tuesday 14th January, 2020 
Ali Khan et al.

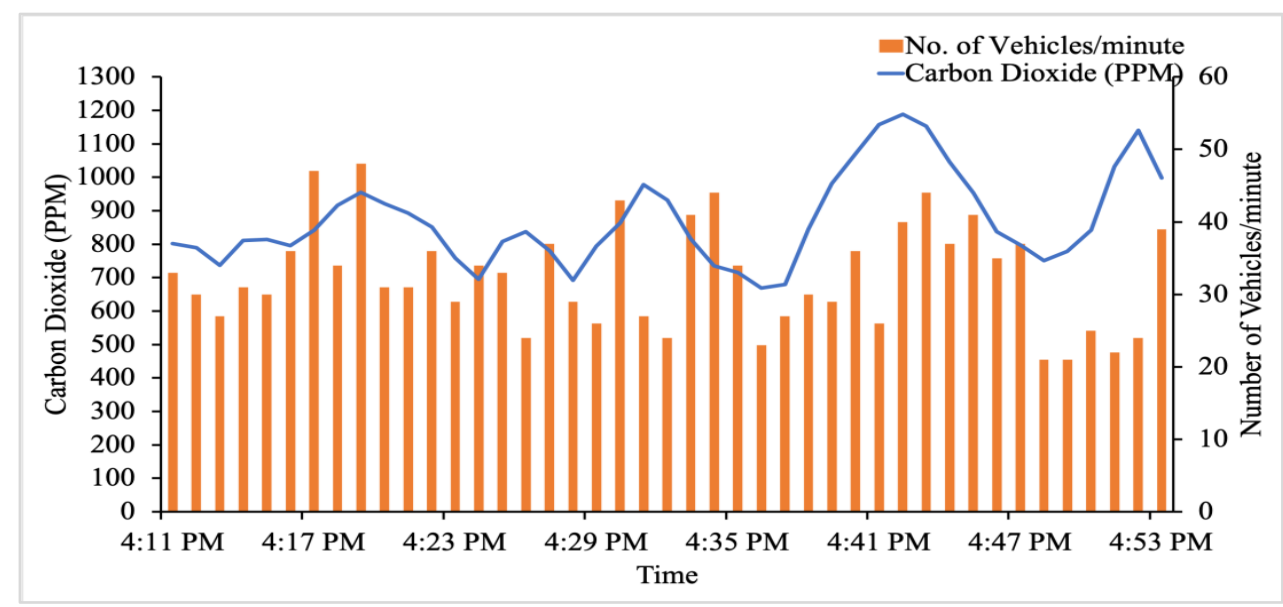

Figure 10 Relationship between vehicle numbers and Carbon Dioxide over 42 min evaluation period on Tuesday $14^{\text {th }}$ January, 2020

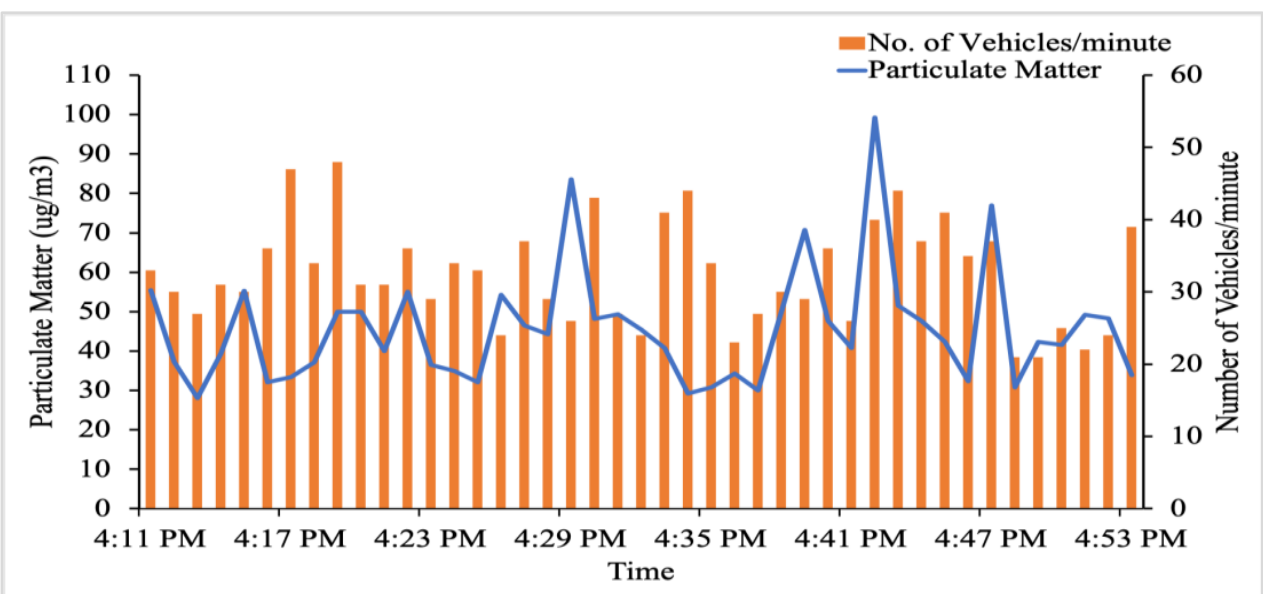

Figure 11 Relationship between vehicle numbers and PM2.5 over a 42 min evaluation period on Tuesday 14th January, 2020

Table 2 Measured traffic flow and road-side vehicular emissions parameters

\begin{tabular}{|c|c|c|c|c|c|c|c|}
\hline Time & Traffic Flow & $\begin{array}{l}\text { Traffic } \\
\text { Density }\end{array}$ & $\begin{array}{l}\text { Average } \\
\text { Headway }\end{array}$ & Time & $\mathbf{P M}_{2.5}$ & $\mathrm{CO}$ & $\mathrm{CO}_{2}$ \\
\hline 4:11 PM & 33 & 1.10 & 1.82 & & 55 & 2.39 & 801 \\
\hline $4: 12$ PM & 30 & 1.00 & 2.00 & & 38 & 2.32 & 788 \\
\hline 4:13 PM & 27 & 0.90 & 2.22 & & 28 & 2.11 & 736 \\
\hline 4:14 PM & 31 & 1.03 & 1.93 & & 39 & 2.47 & 810 \\
\hline 4:15 PM & 30 & 1.00 & 2.00 & & 55 & 2.43 & 813 \\
\hline $4: 16$ PM & 36 & 1.20 & 1.66 & & 32 & 2.34 & 795 \\
\hline 4:17 PM & 47 & 1.57 & 1.27 & & 33 & 2.51 & 842 \\
\hline 4:18 PM & 34 & 1.13 & 1.76 & & 37 & 2.95 & 916 \\
\hline 4:19 PM & 48 & 1.60 & 1.25 & & 50 & 3.04 & 955 \\
\hline 4:20 PM & 31 & 1.03 & 1.93 & & 50 & 2.90 & 921 \\
\hline $4: 21 \mathrm{PM}$ & 31 & 1.03 & 1.93 & & 40 & 2.78 & 892 \\
\hline $4: 22$ PM & 36 & 1.20 & 1.66 & & 55 & 2.58 & 850 \\
\hline $4: 23$ PM & 29 & 0.97 & 2.06 & & 36 & 2.16 & 758 \\
\hline $4: 24$ PM & 34 & 1.13 & 1.76 & & 35 & 1.90 & 695 \\
\hline $4: 25 \mathrm{PM}$ & 33 & 1.10 & 1.81 & & 32 & 2.46 & 808 \\
\hline $4: 26$ PM & 24 & 0.80 & 2.50 & & 54 & 2.56 & 837 \\
\hline 4:27 PM & 37 & 1.23 & 1.62 & & 46 & 2.28 & 780 \\
\hline
\end{tabular}




\begin{tabular}{|c|c|c|c|c|c|c|c|}
\hline Time & Traffic Flow & $\begin{array}{l}\text { Traffic } \\
\text { Density }\end{array}$ & $\begin{array}{l}\text { Average } \\
\text { Headway }\end{array}$ & Time & $\mathbf{P M}_{2.5}$ & $\mathrm{CO}$ & $\mathrm{CO}_{2}$ \\
\hline $4: 28 \mathrm{PM}$ & 29 & 0.97 & 2.06 & & 44 & 1.95 & 691 \\
\hline 4:29 PM & 26 & 0.87 & 2.30 & & 83 & 2.36 & 794 \\
\hline 4:30 PM & 43 & 1.43 & 1.39 & & 48 & 2.67 & 863 \\
\hline $4: 31 \mathrm{PM}$ & 27 & 0.90 & 2.22 & & 49 & 3.16 & 978 \\
\hline 4:32 PM & 24 & 0.80 & 2.50 & & 45 & 3.00 & 931 \\
\hline 4:33 PM & 41 & 1.37 & 1.46 & & 40 & 2.44 & 815 \\
\hline 4:34 PM & 44 & 1.47 & 1.36 & & 29 & 2.09 & 735 \\
\hline $4: 35$ PM & 34 & 1.13 & 1.76 & & 30 & 2.02 & 714 \\
\hline 4:36 PM & 23 & 0.77 & 2.60 & & 34 & 1.83 & 669 \\
\hline 4:37 PM & 27 & 0.90 & 2.22 & & 30 & 1.87 & 678 \\
\hline 4:38 PM & 30 & 1.00 & 2.00 & & 50 & 2.59 & 845 \\
\hline 4:39 PM & 29 & 0.97 & 2.06 & & 70 & 3.20 & 981 \\
\hline 4:40 PM & 36 & 1.20 & 1.66 & & 47 & 3.65 & 1070 \\
\hline 4:41 PM & 26 & 0.87 & 2.30 & & 40 & 4.12 & 1156 \\
\hline $4: 42$ PM & 40 & 1.33 & 1.50 & & 99 & 4.14 & 1187 \\
\hline $4: 43$ PM & 44 & 1.47 & 1.36 & & 51 & 4.01 & 1153 \\
\hline 4:44 PM & 37 & 1.23 & 1.62 & & 47 & 3.53 & 1045 \\
\hline $4: 45 \mathrm{PM}$ & 41 & 1.37 & 1.46 & & 42 & 3.09 & 953 \\
\hline $4: 46$ PM & 35 & 1.17 & 1.71 & & 32 & 2.52 & 837 \\
\hline 4:47 PM & 37 & 1.23 & 1.62 & & 77 & 2.41 & 797 \\
\hline 4:48 PM & 21 & 0.70 & 2.85 & & 30 & 2.19 & 751 \\
\hline 4:49 PM & 21 & 0.70 & 2.85 & & 42 & 2.27 & 778 \\
\hline 4:50 PM & 25 & 0.83 & 2.40 & & 41 & 2.54 & 841 \\
\hline 4:51 PM & 22 & 0.73 & 2.72 & & 49 & 3.48 & 1031 \\
\hline $4: 52$ PM & 24 & 0.80 & 2.50 & & 48 & 3.90 & 1139 \\
\hline $4: 53$ PM & 39 & 1.30 & 1.53 & & 33 & 3.28 & 997 \\
\hline
\end{tabular}

\section{Discussion}

On-road traffic flow characterization is a fundamental building block for provision of any ITS based solutions. As such, varying solutions have been proposed which can be categorized as either intrusive or non-intrusive sensors. However due to inherent limitations, these solutions are incapable to provide full range of traffic flow parameters under all traffic conditions such as under congested and heterogeneous traffic behaviour.

To overcome these limitations, compute vision-based edge computing solutions are emerging as optimum solutions. However, under the constraint of compute resources, proposed solutions in existing literature can provide only traffic count $[10,11]$, count and classification $[4,12,13]$ or count and speed $[8,9$, 14]. However, with incremental improvement in lowcost compute boards and computer vision algorithms, it is expected that edge computing solutions will become capable of providing a full spectrum of traffic flow parameters.

In this work, we have proposed a low-cost solution to measure on-road traffic count, road density and average time headway. The novelty of our work is that in addition to aforementioned traffic flow 191 parameters, roadside vehicular pollutants such as CO, $\mathrm{CO} 2$ and PM2.5 were also measured. Relationships between measured traffic flow parameters and vehicular emissions were established.

\section{Conclusion}

In this work, we have proposed a low-cost and real time cyber physical system for counting vehicles and associated roadside vehicular emissions ( $\mathrm{CO}, \mathrm{CO} 2$, PM2.5). With its edge computing capability, it discards the need of video streaming to servers thus reducing internet data costs. The proposed solution is a step toward exploiting edge computing sensors for implementation of ITS methodologies. Vehicular flow was measured using OpenCV libraries running on RPi using video captured through $\mathrm{Pi}$ camera. Vehicular emissions were sensed through sensors integrated with Arduino Nano. Using Wi-Fi, sensed parameters were transmitted to a free and open source cloud platform 'ThingSpeak' for logging and data analytics. With fabrication cost of $\$ 70$, the proposed solution is both low-cost and is easily installable, and maintainable.

The proposed system was evaluated in a real-world scenario with $86.9 \%$ vehicle counting accuracy. Using sensed data, macroscopic traffic parameters 
such as traffic density, flow and average time headway were calculated. Relationships between traffic flow parameters and associated environmental pollutants were established.

The proposed solution can be employed for identification and assessment of inefficient road sections in terms of traffic flow and environmental costs. Underlying factors for these inefficient road sections range from faulty road design, unsynchronized traffic signals and wrong U-turns taken by drivers. Traffic engineers can then devise strategies to eliminate such inefficiencies from road networks. Moreover, sensed macroscopic traffic flow parameters can be employed for validation and calibration of traffic simulation software and traffic flow mathematical models.

In future, we plan to extend the proposed system with the capability to measure average speed of vehicles. More environmental sensors (such as nitrogen dioxide, ozone and sulphur dioxides) will be integrated for detailed environmental analysis of a particular road section. Multiple of these systems can be installed to form a connected network over a large geographical area. Using harnessed data, a true intelligent transportation system can be achieved.

\section{Acknowledgment}

None.

\section{Conflicts of interest}

This work has been funded by Higher Education Commission, Pakistan to establish a National Centre for Big Data and Cloud Computing at University of Engineering and Technology, Peshawar.

\section{References}

[1] Sohail AM, Khattak KS, Iqbal A, Khan ZH, Ahmad A. Cloud-based detection of road bottlenecks using OBD-II telematics. In international multitopic conference 2019 (pp. 1-7). IEEE.

[2] https://www.who.int/sustainabledevelopment/transport/en/. Accessed 3 January 2020.

[3] https://www.who.int/news-room/factsheets/detail/ambient-(outdoor)-air-quality-and-health. Accessed 3 January 2020.

[4] Gregor D, Cikel K, Arzamendia M, Gregor R. Design and implementation of a counting and differentiation system for vehicles through video processing. International Journal of Computer and Information Engineering. 2016; 10(10):1771-8.

[5] Khan N, Khattak KS, Ullah S, Khan Z. A low-cost IoT based system for environmental monitoring. In international conference on frontiers of information technology (FIT) 2019 (pp. 173-5). IEEE.
[6] Imran W, Khan ZH, Gulliver TA, Khattak KS, Nasir H. A macroscopic traffic model for heterogeneous flow. Chinese Journal of Physics. 2020; 63:419-35.

[7] Khan ZH, Gulliver TA, Azam K, Khattak KS. Macroscopic model on driver physiological and psychological behavior at changes in traffic. Journal of Engineering and Applied Sciences. 2019; 38(2):57-66.

[8] Iszaidy I, Alias A, Ngadiran R, Ahmad RB, Jais MI, Shuhaizar D. Video size comparison for embedded vehicle speed detection \& travel time estimation system by using Raspberry Pi. In international conference on robotics, automation and sciences 2016 (pp. 1-4). IEEE.

[9] McQueen R. Detection and speed estimation of vehicles using resource constrained embedded devices.2018.

[10] Anandhalli M, Baligar VP. A novel approach in realtime vehicle detection and tracking using Raspberry Pi. Alexandria Engineering Journal. 2018; 57(3):1597607.

[11] Wiska R, Alhamidi MR, Habibie N, Wibisono A, Mursanto P, Ramdhan DH, et al. Vehicle traffic monitoring using single camera and embedded systems. In international conference on advanced computer science and information systems 2016 (pp. 117-22). IEEE.

[12] Sorwar T, Azad SB, Hussain SR, Mahmood AI. Realtime vehicle monitoring for traffic surveillance and adaptive change detection using raspberry $\mathrm{Pi}$ camera module. In region 10 humanitarian technology conference 2017 (pp. 481-4). IEEE.

[13] Espinoza FT, Gabriel BG, Barros MJ. Computer vision classifier and platform for automatic counting: more than cars. In second ecuador technical chapters meeting 2017 (pp. 1-6). IEEE.

[14] Jiménez A, García-Díaz V, Anzola J. Design of a system for vehicle traffic estimation for applications on IoT. In proceedings of the 4th multidisciplinary international social networks conference 2017 (pp. 1$6)$.

[15] Khan ZH, Imran W, Gulliver TA, Khattak KS, Wadud Z, Khan AN. An anisotropic traffic model based on driver interaction. IEEE Access. 2020; 8:66799-812.

[16] Iftikhar A, Khan ZH, Gulliver TA, Khattak KS, Khan MA, Ali M, et al. Macroscopic traffic flow characterization at bottlenecks. Civil Engineering Journal. 2020; 6(7):1227-42.

[17] H Khan Z, Imran W, Azeem S, S Khattak K, Gulliver TA, Aslam MS. A macroscopic traffic model based on driver reaction and traffic stimuli. Applied Sciences. 2019; 9(14):1-20.

[18] Khan ZH, Gulliver TA, Khattak KS, Qazi A. A macroscopic traffic model based on reaction velocity. Iranian Journal of Science and Technology, Transactions of Civil Engineering. 2020; 44(1):13950.

[19] https://www.raspberrypi.org/products/raspberry-pi-3model-b/. Accessed 4 January 2020.

[20] Hussain SS, Khattak KS, Khan A, Khan ZH. Cyber physical system for solar energy monitoring. In 
international conference on frontiers of information technology (FIT) 2019. IEEE.

[21] Malik H, Khattak KS, Wiqar T, Khan ZH, Altamimi AB. Low cost internet of things platform for structural health monitoring. In international multitopic conference 2019 (pp. 1-7). IEEE.

[22] Gerlough DL, Huber MJ. Traffic flow theory. 1976.

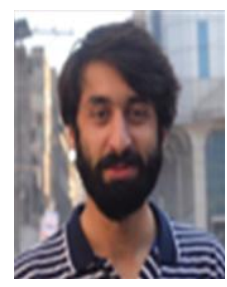

Ali Khan was born in Charsadda, KPK, Pakistan in 1995. He received B.S degree in Computer Systems Engineering from University of Engineering and Technology, Peshawar, in 2018. Currently, he is a research assistant with the National Centre in Big Data and Cloud Computing (NCBC), UET Peshawar from January, 2019. His research interest includes development of systems for Traffic Behaviour Analysis using Embedded Technologies. Email: nangyaly0@gmail.com

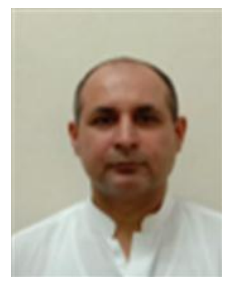

Khurram S. Khattak received his Ph.D. degree from The George Washington University, DC, USA in 2017. He is currently working as Assistant Professor with the Department of Computer Systems Engineering, University of Engineering and Technology Peshawar. His research interests include Intelligent Transportation Systems, Internet of Things (IoT) and Embedded Systems.

Email: khurram.s.khattak@gmail.com

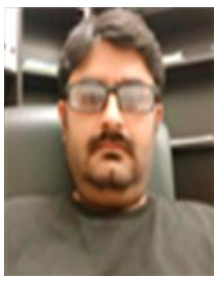

Zawar H. Khan received the Ph.D. degree in Electrical Engineering from the University of Victoria, Victoria, BC, Canada, in 2016. He is currently working as Assistant Professor with the Department of Electrical Engineering, University of Engineering and Technology Peshawar. His research interests include Intelligent Transportation and communication systems.

Email:khanuvic@gmail.com

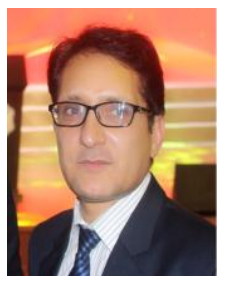

Mushtaq Ahamd Khan has received his $\mathrm{PhD}$ from School of Engineering Mechanics and Materials Hohai University, China. Currently, he is teaching at the University of Engineering and Technology, Mardan, Pakistan. His research interest includes modelling and Numerical simulation, and use of meshless methods in total variation-based image restoration.

Email: mushtaq@uetmardan.edu.pk

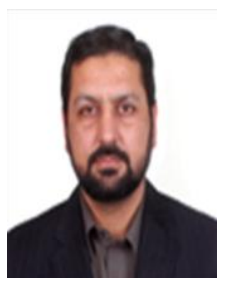

Nasru Minallah received the B.Sc. degree in computer engineering from the University of Engineering and Technology, Peshawar, Pakistan, in 2004 and the M.Sc. degree in computer engineering from Lahore University of Management Sciences, Lahore, Pakistan, in 2006 and Ph.D. degree from the Communications Group, School of Electronics and Computer Science, University of Southampton, Southampton, U.K.in 2010. He is currently working as Associate Professor in Department of Computer Systems Engineering, University of Engineering and Technology Peshawar, Pakistan. His research interests include Image Processing, Remote Sensing, Low-Bit-Rate Video Coding for Wireless Communications.

Email: minallah.nasru@gmail.com 